\title{
An improved formulation of the underground mine scheduling optimisation problem when considering selective mining
}

\author{
SE Terblanche* \\ $\mathrm{A} \mathrm{Bley}^{\dagger}$ \\ Received: 13 February 2014; Revised: 12 June 2014; Accepted: 1 July 2014
}

\begin{abstract}
The use of mixed integer programming is a modelling approach well suited to formulate the mine scheduling optimisation problem for both open pit and underground mining. The resolution applied for discretising the problem, however, has a direct effect on both the level of selectivity that can be applied to improve profitability, as well as the computational feasibility. The proposed model allows for a balance in reducing the resolution used in discretising the underground mine scheduling problem, while maintaining enough detail that will allow the generation of mine production schedules that improve profitability through selective mining. As a secondary contribution, an improved formulation set within a resource production/consumption framework is presented, which can potentially simplify notation used in formulating underground mine scheduling optimisation problems.
\end{abstract}

Key words: Underground mining, scheduling, mixed integer linear programming, aggregation.

\section{Introduction}

The method of extracting valuable minerals from the earth is dependent on the characteristics of the mineral deposits, referred to as the ore body. The two primary methods of mining are open pit mining and underground mining. For the latter, specifically in deep underground gold mining, the ore body is recognised to be a thin sheet or layer-like deposit referred to as a reef, which in some cases are just a few centimetres thick. Depending on its inclination with the surface, a reef could reach depths of up to several kilometres. Open pit mining makes economically more sense when the ore body is more concentrated and closer to the surface.

\footnotetext{
${ }^{*}$ Corresponding author: Centre for Business Mathematics and Informatics, North-West University, Potchefstroom, email: fanie.terblanche@nwu.ac.za

${ }^{\dagger}$ Institute for Mathematics, University of Kassel, Germany 
Irrespective of the mining method, the tasks of planning and executing mining activities are very complex. The optimal use of resources and the timing of production activities could have an enormous effect on the profitability of a mining operation. Furthermore, since the life of a mine can be as long as 60 years, long-term planning is essential to make the right investment choices along the way in order to fully unlock the value of an ore body. The use of mine planning systems to aid decision making is therefore imperative. Specifically, the optimal design of the mine layout and the optimal scheduling of mining activities while taking resource constraints and economic factors into account, are the main driving forces for continued research into realistic mathematical models and efficient solution approaches.

In recent years the use of project scheduling techniques within mining has been applied effectively using readily available project scheduling software tailored for the mining environment. Some of these systems, however, were previously not capable of producing schedules that would maximise a predefined objective function such as Net Present Value (NPV) while satisfying production constraints. Therefore, the term being used in this study namely, mine scheduling optimisation, refers to the generation of an optimal schedule or plan for executing mining activities in future, by taking activity precedence and other side constraints, e.g. capacity constraints, as input.

The use of mixed integer linear programming (MILP) is a modelling approach well suited to formulate the mine scheduling optimisation problem. Compared to open pit applications (for example $[1,2,3,21]$ ), the research published on underground mine scheduling problems is limited. Earlier references to the use of MILP formulations can be found in the publications by Carlyle \& Eaves [4], Rahal et al. [14] and Smith et al. [19], although no model formulations were provided in these papers. MILP formulations for underground mine scheduling problems are presented in the publications by Rubio \& Diering [15] and Schultze \& Zimmermann [18], but without any algorithmic contributions for improving computing times. In the paper by Sarin \& West-Hansen [16], a Benders decomposition approach is followed to improve the solution time of the MILP for small to medium-sized randomly generated problem instances. In the paper by Topal [20], two preprocessing algorithms are presented for reducing the number of binary decision variables in the MILP formulation. Also in an attempt to improve computing times, the approach followed by both Little et al. [10] and Nehring et al. [12] is to aggregate production activities that follow a natural continuous sequence which results in a reduced number of variables. Similarly, the approach by Newman \& Kuchta [13] is to heuristically generate feasible solutions by aggregating time periods. By doing this, improvements on computing times are achieved but to the detriment of optimality. A heuristic approach is presented in Epstein et al. [6] that involves the iterative solving of the linear programming relaxation and the fixing of the binary decision variables. A review of underground mine scheduling optimisation can be be found in Newman et al. [7].

The approach proposed in this paper is related to the work by Little et al. [10], Nehring et al. [12] and Newman \& Kuchta [13], in the sense that the number of variables in the formulation is reduced by introducing a lower time period resolution while maintaining enough information to enable the optimisation model to boost profits through selective mining. As a secondary contribution, a generic formulation of the mine scheduling optimi- 
sation problem cast within a resource production/consumption framework is introduced, with the purpose of simplifying notation.

The model presented in this study is applicable to underground selective mining where the ore grade is highly variable and by selecting out only high grade areas to mine a reduction in cost can be achieved, thus improving profitability. The scope of this work is not limited to a specific mineral.

In the following section an overview of the technical aspects of underground mining is provided. In $\S 3$ a generic model formulation is given that is set within a resource production/consumption framework. The dependence of selective mining on different mining methods is discussed in $\S 4$ and the concept of a mining-method-dependent grade tonnage curve is introduced. In $\S 5$ the newly proposed low resolution resource model with micro selectivity is introduced and in $\S 6$ computational results are provided to show the benefits in applying this new model. A summary and conclusion is provided in $\S 7$.

\section{Some technical aspects of underground mining}

Figure 1 is a simplified illustration of a typical layout of an underground mine. The main vertical tunnel is called the shaft from which horizontal tunnels are excavated to give access to the ore body. The layers of tunnels at different depths are referred to as levels and on each level several smaller tunnels, called raise lines, give access to the valuable minerals along the ore body. Blocks of ore-bearing rock alongside raise lines and in between two levels, are called stoping blocks and are demarcated into smaller pieces to form stoping panels. The activity of excavating stoping panels is referred to as stoping whereas the excavation of tunnels giving access to the ore body is referred to as development. On-reef development refers to the excavation of tunnels (e.g. raise lines) within the ore body with the result that some minerals are also mined out but with a high dilution factor. Off-reef development, on the other hand, refers to the excavation of tunnels through waste rock to give access to the ore body.

Different mining methods can be applied for the excavation of stoping panels. For the purpose of this paper reference is made to two of the most commonly used mining methods in shallow dip reef mining, namely, sequential and pillar mining. For a more complete reference on different mining methods see Hustrulid \& Bullock [8]. Figure 2 illustrates the differences between sequential and pillar mining. For the latter, parts of the stoping block are left behind as pillars for safety purposes, whereas sequential mining would result in clearing out the entire stoping block.

The schedule optimisation problem for underground mining boils down to deciding when to execute a specific mining activity in future, where an activity could relate, for example, to the excavation of part of an underground tunnel per unit time or the placement of machinery that will enable the excavation. This is done for all activities in order to maximise the NPV of the project while taking into account constraints that relate to the physical infrastructure of the mine, like hoisting capacity or other resource constraints such as the available labour force at any given time. The most important set of constraints, however, has to do with activity precedence. That is, except for the very first 


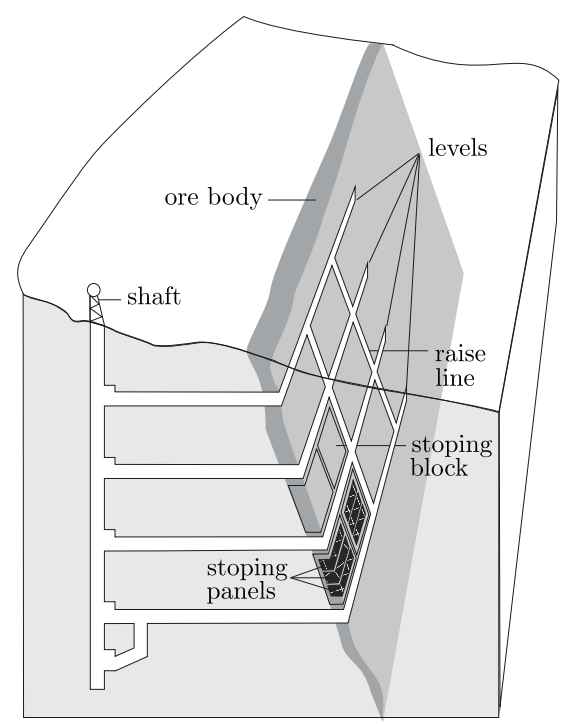

Figure 1: An example of a simplified underground mine layout.
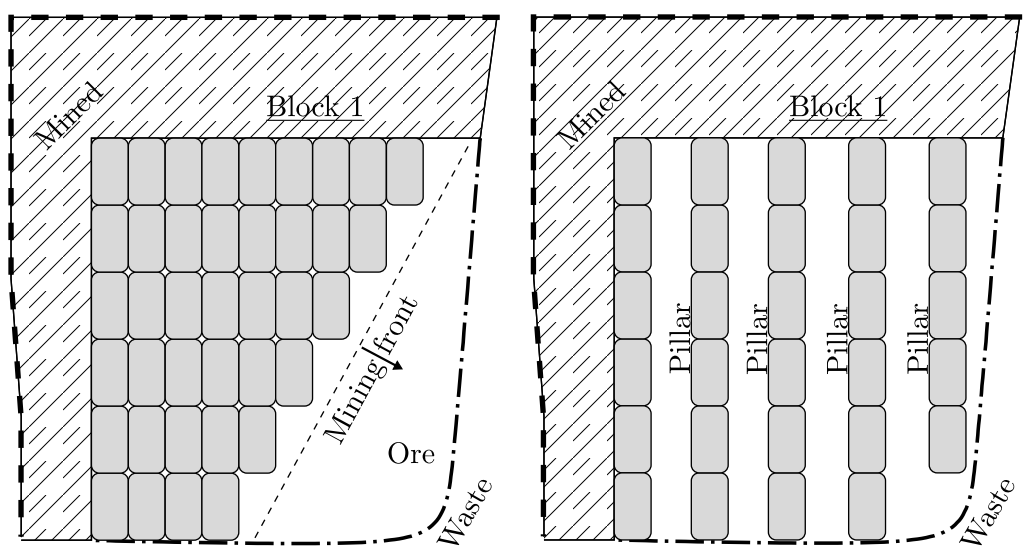

Figure 2: An illustration depicting the differences between sequential and pillar mining (adapted from [9]).

mining activity that would be executed in the mine plan, the execution of all other mining activities will depend on whether their predecessor activities have been completed. For this purpose a precedence graph that is constructed as input to the optimisation model, is used. The structure of such a precedence graph will ultimately depend on the mining method employed. Figure 3 shows the precedence graph (implied by the arrows) for simulating a sequential mining method. Notice that in order to get the step-wise effect of the sequential mining method, the first activity of the second panel (row) is only allowed to start once the second activity of the first panel has been completed, etc.

For a short-term plan the unit of time would typically be a month or even a week, whereas for a long-term plan a unit of time could be for instance a year. Clearly, with an increase in time period resolution an increase in computing times could be expected due to an increase in the number of variables that have to be considered. 


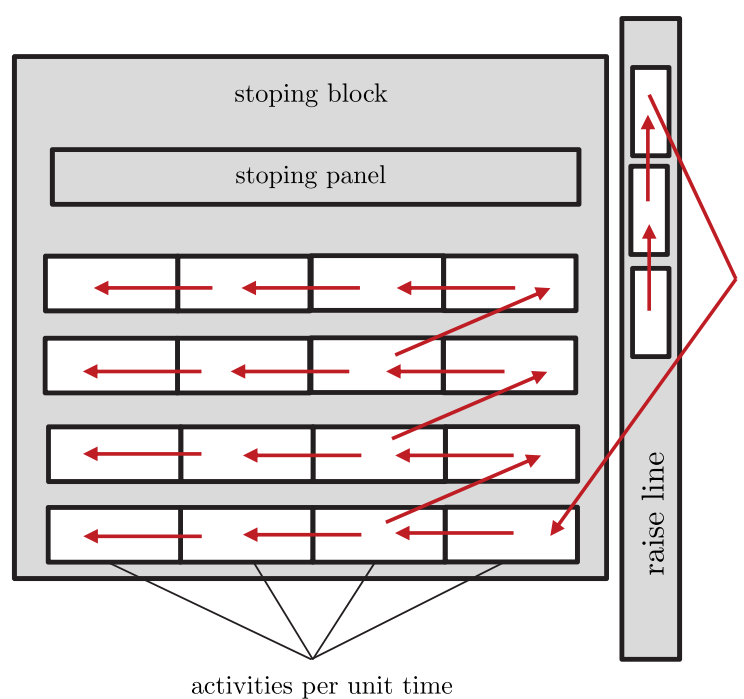

Figure 3: Precedence graph used for simulating a sequential mining method.

In this paper it is not the intention to model the underground mine production problem into the finest detail, nor is the focus on specific mining methods that would result in very specific side constraints. The objective of this study is to provide a generic formulation that has the primary constraint ingredients such as activity precedence, but that gives a good trade-off between reducing time period resolution and maintaining enough information that would allow selective mining based on the grade variability of the ore body.

\section{Model formulation and notation}

The underground mine scheduling optimisation problem is formulated in this paper as a MILP. In the section below the proposed formulation is set within a generic resource production/consumption framework in an attempt to simplify notation. Thereafter, amendments to the formulation are proposed that will result in a reduction in the number of variables, but at the same time will maintain enough information to allow the simulation of selective mining.

\subsection{The resource model}

The basic idea of the resource production/consumption framework is that each activity is viewed as either being resource consuming or resource producing or both. By creating a set of different resources which are either produced (minerals) or consumed (labour hours), the notation used for the problem formulation can be simplified.

- Let $\mathcal{R}$ denote the set of resources. A potential set of resources could include the number of tonnes from stoping production, the volume of minerals produced through processing the ore, the amount of explosives consumed for blasting, the number of labour hours consumed for excavation, etc. Note that a resource $r \in \mathcal{R}$ is not an actual numerical value but rather the name of a specific resource. 
- Let $\mathcal{A}$ denote the index set of all activities. An activity $a \in \mathcal{A}$ could relate to the excavation of part of an underground tunnel per unit time or the placement of machinery that will enable the excavation, etc. Note that the size of the set $\mathcal{A}$ is a function of the time period resolution used in the discretisation of the problem. For instance, for a high resolution discretisation where each activity $a \in \mathcal{A}$ relates to a task being performed within, say, a single week, the size of $\mathcal{A}$ would be considerably larger compared to a lower resolution discretisation where each activity $a \in \mathcal{A}$ relates to a task being performed within, say, a month.

- Let $(a-1)$ denote the predecessor activity of $a \in \mathcal{A}$. For the purpose of simplifying the presentation of the approach in this article, it is assumed that each activity will only have a single predecessor.

- Let $\mathcal{A}(r)$ denote the set of activities that either consumes or produces the resource $r \in \mathcal{R}$.

- Let $\delta_{r a} \geq 0$ be a numerical value for the quantity of resource $r \in \mathcal{R}$ being produced/consumed by activity $a \in \mathcal{A}$. Note that this quantity is dependent on the time period resolution and would be determined as part of a preprocessing step. If a constant rate at which activity $a \in \mathcal{A}$ is being performed, is considered, an increase in the time period size would result in a linear increase in $\delta_{r a}$.

- Let $\mathcal{T}=\{1,2, \ldots,|\mathcal{T}|\}$ denote the time period indices.

- Let $(t-1)$ denote the predecessor time period for $t \in \mathcal{T}$.

- Let $c_{r t}$ be the value per unit of consuming/producing a resource $r \in \mathcal{R}$ in a time period $t \in \mathcal{T}$. Note that the coefficient $c_{r t}$ could either be negative or positive, depending on the type of resource. For instance, for a maximisation problem, the coefficient associated with a resource that denotes the tonnes of rock from stoping production would be negative, since the production of the resource would incur costs. However, the coefficient associated with the volume of minerals, would be treated as positive since revenue is incurred by the production of this resource.

- Let $p$ denote the time period resolution parameter expressed as the number of months contained within a single period. That is, if $p=1$, a monthly calendar is considered, with each period $t \in \mathcal{T}$ being exactly one month and if, for example, $p=12$, then an annual calendar is considered where each period $t \in \mathcal{T}$ is taken to be one year.

- Let $U_{r t}$ be the upper limit on the quantity of resource $r \in \mathcal{R}$ that may be consumed/produced for time period $t \in \mathcal{T}$ expressed as a value per month. Generalisation of $U_{r t}$ to other time period resolutions is obtained by multiplying with the parameter $p$.

- Let $L_{r t}$ be the lower limit on the quantity of resource $r \in \mathcal{R}$ that may be consumed/produced for time period $t \in \mathcal{T}$ expressed as a value per month. Generalisation of $L_{r t}$ to other time period resolutions is obtained by multiplying with the parameter $p$.

- Let $d^{(p)}$ be the effective NPV rate, for a period of size $p$, at which future cash-flows will be discounted with. This will ensure that the discounting is done according to the correct period sizes when schedules generated with different time period resolutions are compared.

As a result of formulating the problem within a resource production/consumption framework, it is not necessary to distinguish between different types of mining activities when 
defining the decision variables. The use of the parameters $\delta_{r a}$ will translate the decision to execute the activity $a \in \mathcal{A}$ into a measurable quantity related to the resource $r \in \mathcal{R}$. Consequently, if the variable $z_{a t} \in\{0,1\}$ is defined to take on a value of one if the mining activity $a$ is scheduled to be executed in time period $t$. A binary integer programming problem (referred to as the Resource based Mine Scheduling Optimization Problem (RMSOP)), may then be formulated with the objective to

$$
\begin{array}{cc}
\operatorname{maximise} \sum_{t \in \mathcal{T}}\left(1+d^{(p)}\right)^{-t} \sum_{r \in \mathcal{R}} \sum_{a \in \mathcal{A}(r)} c_{r t} \delta_{r a} z_{a t}, & \\
\text { subject to } z_{a t} \leq \sum_{\substack{k \in \mathcal{T} \\
k<t}} z_{(a-1) k} & a \in \mathcal{A}, t \in \mathcal{T}, \\
\sum_{a \in \mathcal{A}} z_{a t} \leq 1 & t \in \mathcal{T}, \\
p L_{r t} \leq \sum_{a \in \mathcal{A}(r)} \delta_{r a} z_{a t} \leq p U_{r t} & r \in \mathcal{R}, t \in \mathcal{T} .
\end{array}
$$

The objective function (1) will maximise NPV at a discount rate of $d^{p}$, provided that appropriate cost coefficients $c_{r t}$ are defined for each of the relevant resources $r \in \mathcal{R}$ and for each time period $t \in \mathcal{T}$. Constraint set (2) enforces the precedence relationship. An activity $a \in \mathcal{A}$ can only be performed once its predecessor $(a-1) \in \mathcal{A}$ has been completed. Constraint set (3) will only allow an activity to be scheduled once. Note that the inequality sign in constraint set (3) enables the model to allow selective mining since an activity may not to be scheduled at all. Constraint set (4) provides upper and lower limits on the consumption and production of resources respectively. From an implementation point of view, the benefit of having these generic constraints is that it is easy to add limits on resource consumption/production by simply adding the said resources to $\mathcal{R}$ and by specifying the limits $L_{r t}$ and $U_{r t}$, without having to explicitly define additional constraint for the model.

An important property of the RMSOP formulation is that computational complexity worsens with an increase in the time period resolution. On the positive side, by increasing the time period resolution, selectivity is improved due to more detail that becomes available by discretising the problem into smaller activity pieces. That is, instead of scheduling a monthly activity for mining with a diluted grade, the period size can be reduced to a week and only part of the same activity that coincides with a higher grade can be scheduled, leaving behind the low grade portion. Therefore, a clear trade-off exist between more information that will allow improved selective mining versus a worsening of computing times as a result of increasing time period resolution.

\subsection{A preprocessing step for variable reduction}

The preprocessing performed on the RMSOP in order to reduce the number of variables is based on the approach described in the publications by Newman \& Kuchta [13] and Topal [20]. The basic idea is that each activity has an earliest possible starting time 
and a latest possible finishing time. This is determined by the chain of predecessors and successors to the activity. For example, if an activity $a_{4} \in \mathcal{A}$ has the chain of predecessor activities $\left\{a_{3}, a_{2}, a_{1}\right\}$ where $a_{3}$ is the immediate predecessor of $a_{4}, a_{2}$ is the immediate predecessor of $a_{3}$ and $a_{1}$ is the immediate predecessor of $a_{2}$, then activity $a_{4}$ can start at the earliest in the fourth period if each of the predecessors in the chain is scheduled to be performed in each of the preceding time periods. By having an earliest starting time $t_{0 a} \in \mathcal{T}$ and a latest finishing time $t_{1 a} \in \mathcal{T}$ for each activity $a \in \mathcal{A}$, all the variables $z_{a t}$ for all $t<t_{0 a}$ and $t>t_{1 a}$ can be removed from the problem formulation.

\section{Selectivity and mining methods}

The application of selectivity in mining is motivated by high variability in grade. Leaving low grade stoping areas behind leads to improved profitability. However, the mining method employed could limit the level of selectivity applied. By considering the allowable sequencing for sequential mining depicted in Figure 3, it is clear that selectivity is spatially constrained. That is, because the stoping activities are not scheduled in the lower-right corner, it is necessary to leave behind the rest of the stoping block. Consequently, benefits will mostly be gained from selectivity within a sequential mining context when stoping activities in the upper-left area of a stoping block having a lower grade are left behind and not scheduled to be mined.

\begin{tabular}{|c|c|c|c|c|c|c|c|c|c|c|c|c|}
\hline \multirow[b]{2}{*}{ Period } & \multicolumn{10}{|c|}{ Panels } & \multirow{2}{*}{$\begin{array}{r}\text { Cumulative } \\
\text { content }(\mathrm{kg})\end{array}$} & \multirow{2}{*}{$\begin{array}{r}\text { Cumulative } \\
\text { tonnes }(\mathrm{t})\end{array}$} \\
\hline & 1 & 2 & 3 & 4 & 5 & 6 & 7 & 8 & 9 & 10 & & \\
\hline 1 & 153.3 & & & & & & & & & & 153.3 & 12831 \\
\hline 2 & 193.9 & 119.7 & & & & & & & & & 466.9 & 51911 \\
\hline 3 & 181 & 99.6 & 153.8 & & & & & & & & 901.3 & 127350 \\
\hline 4 & 112.5 & 41 & 128.9 & 76 & & & & & & & 1259.7 & 232786 \\
\hline 5 & 217.2 & 76.4 & 151 & 82.8 & 44.9 & & & & & & 1832.0 & 386125 \\
\hline 6 & 78.4 & 150.3 & 114.6 & 10.5 & 16.6 & 55.1 & & & & & 2257.5 & 575078 \\
\hline 7 & 22 & 118.9 & 50.3 & 116.1 & 1 & 1 & 115.2 & & & & 2682.0 & 799561 \\
\hline 8 & 83 & 78.5 & 35 & 54 & 35.5 & 1 & 4.2 & 1 & & & 2974.2 & 1048502 \\
\hline 9 & 62.3 & 52 & 43 & 39 & 34 & 35 & 32.2 & 35.9 & 32.2 & & 3339.8 & 1328043 \\
\hline 10 & 42.3 & 43.1 & 38.1 & 29 & 45 & 52.1 & 33.8 & 72.2 & 27 & 54 & 3776.4 & 1644127 \\
\hline 11 & 23.3 & 44.6 & 3.6 & 51.2 & 1 & 34 & 12.8 & 53 & 19 & 39 & 4057.9 & 1983774 \\
\hline 12 & 1 & 4.7 & 1 & 32 & 15 & 24 & 1 & 20.6 & 23 & 25 & 4205.2 & 2335749 \\
\hline
\end{tabular}

Table 1: Example data for creating a spatial grade tonnage curve for monthly stoping activities when considering a sequential mining method.

The concept of a grade tonnage curve which has been used for decades by mining practitioners is central to the practice of selective mining. It represents the relationship between the level of selectivity and the expected grade. This relationship is represented as a cumulative graph, obtained by ranking demarcated ore blocks according to their grade in descending order, cumulatively adding their volume and plotting the cumulative volume versus the average grade over the accumulated blocks. This method does not take the mining method into account and assumes the effect of cherry picking whereby high grade portions can be removed even though the mining method may not permit it. For the purpose of this paper, a mining-method-dependent grade tonnage curve (henceforth MMD grade tonnage curve) is therefore used, which is surprisingly not very often used in mine planning. The only reference that could be found on the topic is the paper by Silva \& 
Soares [17]. The construction of an MMD grade tonnage curve is illustrated by means of example data listed in Table 1, where a single stoping block comprising 10 stoping panels being mined over a period of 12 months, is considered.

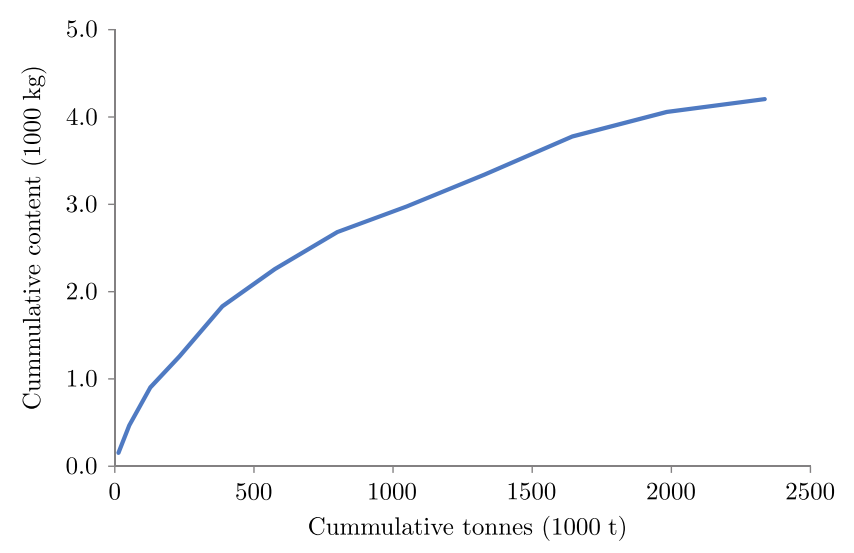

Figure 4: An MMD grade tonnage curve created with example data from Table 1.

The entries for the matrix formed by the period versus panel intersection, reflect the mineral content obtained by mining part of a panel in a specific time period. That is, the entries correspond to the mineral content associated with each stoping activity. Furthermore, from Table 1 it is clear that mining of the second panel only starts one period after the first panel, due to the sequential mining method adopted. The column on the right-hand side of Table 1, labelled Cumulative content $(\mathrm{kg})$, is obtained by summing the mineral content for each period over all the panels. Since it is assumed for this example that all stoping activities are of the same dimension, i.e. they all correspond to the excavation of exactly the same tonnages per time period, the column on the far right-hand side labelled Cumulative tonnages $(t)$ is obtained by summing the tonnages for each period over all the panels.

The MMD grade tonnage curve depicted in Figure 4 is obtained by plotting the columns Cumulative tonnages ( $\mathrm{t}$ ) against Cumulative content $(\mathrm{kg})$. From the graph there is a clear change in the slope of the function as mining advances through the stoping block, implying that profitability could be improved by leaving behind a portion of the stoping block due to a decline in grade. The benefit of having such a summarised view of the stoping block is that instead of having variables that relate to each of the activities that could be scheduled in one of many alternative periods, a single variable that will denote the extraction of part of the stoping block could be introduced.

\section{A low resolution resource model with micro selectivity}

The use of an MMD grade tonnage curve facilitates the formulation of the mine scheduling optimisation problem with a lower time period resolution, resulting in a reduced number of activities and eventually a reduced number of variables. Since the MMD grade tonnage curve is constructed out of a higher resolution discretised problem, enough information is taken into account to allow selectivity on a micro level. 
The approach is thus to first discretise the problem into, say, monthly activities. The monthly stoping activities are then grouped by stoping blocks and an MMD grade tonnage curve is then constructed for each stoping block by using the monthly stoping activities as was illustrated in Table 1. It should be noted that the monthly discretisation should be performed without taking any capacity constraints into account such that the only factors influencing the shape of the MMD grade tonnage curves would be the mining method and the grade distribution. The next step is to discretise the problem according to a lower time period resolution, e.g. by using annual activities. These activities will be used as the set $\mathcal{A}$ in the problem formulation. The last step is then to associate the annual stoping activities with the stoping blocks such that several annual stoping activities can be mapped to a single MMD grade tonnage curve. Note that in order to maintain linearity each MMD grade tonnage curve is approximated with a piece-wise linear approximation. Figure 5 illustrates the approximation of an MMD grade tonnage curve using three line segments. The points $\left(x_{0}, y_{0}\right),\left(x_{1}, y_{1}\right),\left(x_{2}, y_{2}\right)$ and $\left(x_{3}, y_{3}\right)$ are called the knots of the piece-wise linear function. In order to formulate this into the model the following notation is required.

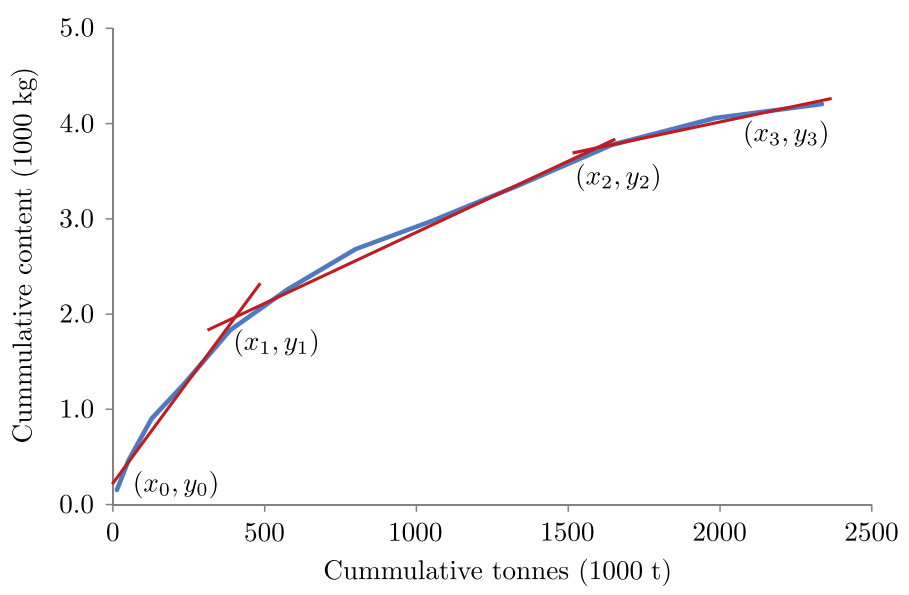

Figure 5: A linear approximation of an MMD grade tonnage curve.

- Let $\mathcal{B}$ denote the set of all stoping blocks.

- Let $\mathcal{A}(b)$ denote the set of stoping activities associated with a stoping block $b \in \mathcal{B}$.

- Let the points $\left(x_{b i}, y_{b i}\right), i \in \mathcal{I}=\{0,1,2, \ldots, N-1\}$ be the knots for the piece-wise linear approximation of the MMD grade tonnage curve associated with the stoping block $b \in \mathcal{B}$.

The auxiliary variables $x_{b} \geq 0$ are introduced to incorporate the MMD grade tonnage curve into the problem formulation, denoting the tonnes of the stoping block $b \in \mathcal{B}$ that will be extracted and $y_{b} \geq 0$ denoting the corresponding mineral content that will be produced according to the MMD grade tonnage curve associated with stoping block $b \in \mathcal{B}$. To account for the mineral content and tonnes extracted per period, the variables $x_{a t} \geq 0$ and $y_{a t} \geq 0$ are defined such that $x_{b}=\sum_{a \in \mathcal{A}(b)} \sum_{t \in \mathcal{T}} x_{a t}$ and $y_{b}=\sum_{a \in \mathcal{A}(b)} \sum_{t \in \mathcal{T}} y_{a t}$ for all $b \in \mathcal{B}$. To align these newly introduced auxiliary variables with the proposed generic resource based framework, the subset of resources $\mathcal{R}^{s} \subseteq \mathcal{R}$ is introduced which is exclusively being associated with stoping activities. For the subsequent formulations, $r_{1} \in \mathcal{R}^{s}$ 
denotes the resource associated with the kilograms of minerals produced and $r_{2} \in \mathcal{R}^{s}$ the resource associated with the tonnes of stoping rock produced. By associating the variable $y_{a t}$ with the resource $r_{1}$ and the variable $x_{a t}$ with the resource $r_{2}$, it is possible to correctly calculate the revenue and costs respectively. These associations are established through the use of the quantities $\delta_{r_{1} a}$ and $\delta_{r_{2} a}$ denoting the actual kilograms of minerals produced and the tonnes of stoping rock produced by executing activity $a \in \mathcal{A}$, respectively. The coefficients $c_{r_{1} t}$ is defined to reflect the (positive) mineral prices and $c_{r_{2}}$ to reflect the (negative) stoping production cost, for all $t \in \mathcal{T}$.

The modelling technique employed here to represent the MMD grade tonnage curves as piece-wise linear approximations (see e.g. [5] and [11]), requires the introduction of the auxiliary variables $\lambda_{i} \geq 0$, with $i \in \mathcal{I}$ and $l_{j} \in\{0,1\}$, with $j \in \mathcal{I} \backslash\{0\}=\{1,2, \ldots, N-1\}$. The latter is for selecting the most appropriate line segment for local approximation with respect to the objective function, whereas the former is needed to express the decision variables $x_{b}$ and $y_{b}$ as convex combinations of the knots $\left(x_{b i}, y_{b i}\right), i=0,1,2, \ldots, N-1$.

The formulation of the RMSOP is adapted to obtain the Low Resolution model with Micro Selectivity (LRMS). The objective then becomes to

$$
\operatorname{maximise} \sum_{t \in \mathcal{T}}\left(1+d^{(p)}\right)^{-t}\left\{\sum_{r \in \mathcal{R} \backslash \mathcal{R}^{s}} \sum_{a \in \mathcal{A}(r)} c_{r t} \delta_{r a} z_{a t}+\sum_{\substack{a \in \mathcal{A}\left(r_{1}\right) \\ r_{1} \in \mathcal{R}^{s}}} c_{r_{1} t} y_{a t}+\sum_{\substack{a \in \mathcal{A}\left(r_{2}\right) \\ r_{2} \in \mathcal{R}^{s}}} c_{r_{2} t} x_{a t}\right\},
$$

$$
\begin{array}{rlrl}
\text { subject to } z_{a t} \leq \sum_{\substack{k \in \mathcal{T} \\
k<t}} z_{(a-1) k} & a \in \mathcal{A}, t \in \mathcal{T}, \\
\sum_{a \in \mathcal{A}} z_{a t} \leq 1 & t \in \mathcal{T}, \\
p L_{r t} \leq \sum_{a \in \mathcal{A}(r)} \delta_{r a} z_{a t} \leq p U_{r t} & r \in \mathcal{R}, t \in \mathcal{T}, \\
y_{a t} \leq \delta_{r_{1} a} \sum_{k \in \mathcal{T}} z_{a k} & b \in \mathcal{B}, a \in \mathcal{A}(b), t \in \mathcal{T}, r_{1} \in \mathcal{R}^{s}, \\
x_{a t} \leq \delta_{r_{2} a} \sum_{k \in \mathcal{T}} z_{a k} & b \in \mathcal{B}, a \in \mathcal{A}(b), t \in \mathcal{T}, r_{2} \in \mathcal{R}^{s}, \\
\delta_{r_{1}(a-1)} z_{a t} \leq \sum_{k \in \mathcal{T}} y_{(a-1) k} & b \in \mathcal{B}, a \in \mathcal{A}(b),(a-1) \in \mathcal{A}(b), \\
x_{b}=\sum_{a \in \mathcal{A}(b)} \sum_{t \in \mathcal{T}} x_{a t} & t \in \mathcal{T}, r_{1} \in \mathcal{R}{ }^{s} \\
y_{b}=\sum_{a \in \mathcal{A}(b)} \sum_{t \in \mathcal{T}} y_{a t} & b \in \mathcal{B},
\end{array}
$$




$$
\begin{array}{rlrl}
x_{b} & =\sum_{i \in \mathcal{I}} \lambda_{i b} x_{i b} & & b \in \mathcal{B}, \\
y_{b} & =\sum_{i \in \mathcal{I}} \lambda_{i b} y_{i b} & & b \in \mathcal{B}, \\
\sum_{i \in \mathcal{I}} \lambda_{i b} & =1 & & b \in \mathcal{B}, \\
\lambda_{0 b} & \leq l_{1 b} & b & \in \mathcal{B}, \\
\lambda_{i b} & \leq l_{i b}+l_{(i+1) b} & b & \in \mathcal{B}, i \in \mathcal{I} \backslash\{0, N-1\}, \\
\lambda_{(N-1) b} & \leq l_{(N-1) b} & b & \in \mathcal{B} .
\end{array}
$$

The objective function (5) comprises the costs associated with non-stoping activities indexed through the resource set $\mathcal{R} \backslash \mathcal{R}^{s}$ and revenues associated with the stoping activities indexed through the resource $r_{1} \in \mathcal{R}^{s}\left(r_{2} \in \mathcal{R}^{s}\right)$. Note that the second and third terms in the objective function do not need the multipliers $\delta_{r a}$ for the variables $y_{a t}$ and $x_{a t}$, since these variables already reflects the mineral content and stoping tonnages respectively, obtained through the MMD grade tonnage curve relationship.

Constraint sets (6), (7) and (8) are exactly the same as constraint sets (2), (3) and (4) from the RMSOP and will have the same purpose within the LRMS formulation. Constraint set (9) is responsible for allowing the mineral content variables $y_{a t}$ to take on a value only if the corresponding activity has been scheduled to be executed. Furthermore, these constraints will also limit the variables $y_{a t}$ to a level of $\delta_{r_{1} a}$ to maintain the proportionality of the activity $a \in \mathcal{A}(b)$ with respect to the stoping block $b$. Analogous to this constraint set (10) governs the values that the variables $x_{a t}$ may take on relative to the activity variables $z_{a t}$. The purpose of the constraint set (11) is to allow an activity to be executed only if its predecessor has been completed, i.e. if the mineral content variable $y_{(a-1) t}$ has reached its limit $\delta_{r_{1}(a-1)}$. Note that these constraints require that $\delta_{r_{1}(a-1)}$ be strictly positive to prevent them from being fulfilled trivially. The assumption, therefore, is that each aggregated activity $a \in \mathcal{A}(b)$ would always be a mineral producing activity within the block $b$. Constraint sets (12) and (13) aggregate the time dependent variables $x_{a t}$ and $y_{a t}$ to the stoping block totals $x_{b}$ and $y_{b}$. Constraint sets (15) and (16) in turn express these stoping block totals as convex combinations of the piece-wise linearisation knots of the MMD grade tonnage curve. Evidently, constraint set (17) maintains the necessary convexity conditions and constraint sets (18), (19) and (20) are responsible for enabling the appropriate convexity variable $\lambda_{i b}$ to take on a value based on the selection of a specific line segment $l_{i b}$.

An important feature of the LRMS model, compared to the RMSOP model, is that precedence relationships might be distorted during aggregation. Specifically, in cases where branching occurs within the precedence of a high resolution discretisation, it might be that for the corresponding low resolution discretisation the branches are now either allowed to be executed during the same period as its predecessor or much later after the total aggregation has been completed, depending on the aggregation policy applied. 


\section{Empirical results}

Empirical tests were performed to determine whether the LRMS model would improve on computing times by considering a lower time period resolution, while enabling micro selectivity through the use of MMD grade tonnage curves. The data used were generated randomly for a range of problem instances with a biased grade distribution that will favour selective mining. That is, the grade tonnage curves were created to reflect a decrease in grade as excavation advances through the stoping block over time making it economically more attractive to be selective. Selective mining may, however, be less attractive with an increase in mineral price (provided constant costs) due to improved profit margins. The interplay between the level of selectivity and increasing mineral prices were also examined as part of this empirical study.

\begin{tabular}{|c|c|c|c|c|c|c|c|c|c|c|c|}
\hline \multicolumn{2}{|c|}{ Problem instance } & \multicolumn{2}{|c|}{ Mineral price $(\times 1)$} & \multicolumn{2}{|c|}{ Mineral price $(\times 1.25)$} & \multicolumn{2}{|c|}{ Mineral price $(\times 1.5)$} & \multicolumn{2}{|c|}{ Mineral price $(\times 1.75)$} & \multicolumn{2}{|c|}{ Mineral price $(\times 2)$} \\
\hline Resolution & \#Blocks & NPV & gap (\%) & NPV & gap (\%) & NPV & gap (\%) & NPV & gap (\%) & NPV & gap (\%) \\
\hline \multirow{8}{*}{$\begin{array}{l}\text { Monthly } \\
\text { (RMSOP) }\end{array}$} & 10 & $9 \times 10^{6}$ & 62.7 & $5.7 \times 10^{7}$ & 19.3 & $1.3 \times 10^{8}$ & 15.0 & $2.2 \times 10^{8}$ & 10.5 & $9.9 \times 10^{9}$ & 0.11 \\
\hline & 20 & $2 \times 10^{7}$ & $>100$ & $1.2 \times 10^{8}$ & 32.6 & $2.6 \times 10^{8}$ & 19.9 & $4.4 \times 10^{8}$ & 14.8 & $1.9 \times 10^{10}$ & 0.29 \\
\hline & 30 & 0 & $>100$ & $1.6 \times 10^{8}$ & 48.7 & $3.8 \times 10^{8}$ & 23.4 & $6.3 \times 10^{8}$ & 17.2 & $2.7 \times 10^{10}$ & 0.32 \\
\hline & 40 & 0 & $>100$ & $1.5 \times 10^{8}$ & $>100$ & $4.8 \times 10^{8}$ & 25.1 & $8.1 \times 10^{8}$ & 17.8 & $3.5 \times 10^{10}$ & 0.35 \\
\hline & 50 & 0 & $>100$ & $3.1 \times 10^{5}$ & $>100$ & $5.8 \times 10^{8}$ & 24.9 & $1.1 \times 10^{9}$ & 19.3 & $4.2 \times 10^{10}$ & 0.46 \\
\hline & 60 & 一 & - & - & - & - & - & - & - & - & - \\
\hline & 100 & - & - & - & - & - & - & - & - & - & - \\
\hline & 200 & - & - & - & - & - & - & - & - & - & - \\
\hline \multirow{8}{*}{$\begin{array}{l}\text { Annually } \\
\text { (RMSOP) }\end{array}$} & 10 & 0 & 0 & $7.0 \times 10^{7}$ & 0 & $6.1 \times 10^{7}$ & 0 & $1.4 \times 10^{8}$ & 0 & $2.2 \times 10^{8}$ & 0 \\
\hline & 20 & 0 & 0 & $1.8 \times 10^{7}$ & 0 & $1.3 \times 10^{8}$ & 0 & $2.8 \times 10^{8}$ & 0 & $4.4 \times 10^{8}$ & 0 \\
\hline & 30 & 0 & 0 & $2.8 \times 10^{7}$ & 0 & $1.9 \times 10^{8}$ & 0 & $4.0 \times 10^{8}$ & 0 & $6.3 \times 10^{8}$ & 0 \\
\hline & 40 & 0 & 0 & $3.3 \times 10^{7}$ & 0 & $2.4 \times 10^{8}$ & 0 & $5.1 \times 10^{8}$ & 0 & $8.0 \times 10^{8}$ & 0 \\
\hline & 50 & 0 & 0 & $4.0 \times 10^{7}$ & 0 & $2.8 \times 10^{8}$ & 0 & $6.1 \times 10^{8}$ & 0 & $9.5 \times 10^{8}$ & 0 \\
\hline & 60 & 0 & 0 & $4.6 \times 10^{7}$ & 0 & $3.2 \times 10^{8}$ & 0 & $7.0 \times 10^{8}$ & 0 & $1.1 \times 10^{9}$ & 0 \\
\hline & 100 & 0 & 0 & $6.7 \times 10^{7}$ & 0 & $4.5 \times 10^{8}$ & 0 & $9.8 \times 10^{8}$ & 0 & $1.5 \times 10^{9}$ & 0 \\
\hline & 200 & 0 & 0 & $1.0 \times 10^{8}$ & 0 & $6.4 \times 10^{8}$ & 0.8 & $1.4 \times 10^{9}$ & 0.3 & $2.1 \times 10^{9}$ & 0.20 \\
\hline \multirow{8}{*}{$\begin{array}{l}\text { Annually with } \\
\text { MMD grade } \\
\text { tonnage curve } \\
\text { (LRMS) }\end{array}$} & 10 & $2.0 \times 10^{7}$ & 2.8 & $7.4 \times 10^{7}$ & 1.8 & $1.3 \times 10^{8}$ & 2.5 & $1.9 \times 10^{8}$ & 1.1 & $2.7 \times 10^{8}$ & 0.70 \\
\hline & 20 & $6.0 \times 10^{7}$ & 12.9 & $1.6 \times 10^{8}$ & 6.6 & $2.7 \times 10^{8}$ & 5.4 & $4.0 \times 10^{8}$ & 2.4 & $5.6 \times 10^{8}$ & 1.30 \\
\hline & 30 & $1.0 \times 10^{8}$ & 23.5 & $2.5 \times 10^{8}$ & 11.6 & $4.1 \times 10^{8}$ & 8.4 & $6.0 \times 10^{8}$ & 4.2 & $8.2 \times 10^{8}$ & 2.30 \\
\hline & 40 & $2.0 \times 10^{8}$ & 25.8 & $3.4 \times 10^{8}$ & 14.1 & $5.4 \times 10^{8}$ & 11.6 & $7.8 \times 10^{8}$ & 7.0 & $1.1 \times 10^{9}$ & 3.80 \\
\hline & 50 & $2.0 \times 10^{8}$ & 29.2 & $4.3 \times 10^{8}$ & 17.5 & $6.7 \times 10^{8}$ & 14.5 & $9.6 \times 10^{8}$ & 9.8 & $1.3 \times 10^{9}$ & 5.60 \\
\hline & 60 & $3.0 \times 10^{8}$ & 28.8 & $5.3 \times 10^{8}$ & 19.4 & $8.0 \times 10^{8}$ & 17.0 & $1.1 \times 10^{9}$ & 11.7 & $1.5 \times 10^{9}$ & 7.60 \\
\hline & 100 & $5.0 \times 10^{8}$ & 33.6 & $8.9 \times 10^{8}$ & 29.6 & $1.3 \times 10^{9}$ & 27.7 & $1.8 \times 10^{9}$ & 20.5 & $2.3 \times 10^{9}$ & 14.30 \\
\hline & 200 & $1.0 \times 10^{9}$ & 52.4 & $1.6 \times 10^{9}$ & 51.9 & $2.2 \times 10^{9}$ & 44.2 & $2.8 \times 10^{9}$ & 37.9 & $3.6 \times 10^{9}$ & 28.80 \\
\hline
\end{tabular}

Table 2: Objective function values and integrality gaps for solving the mine scheduling optimisation problem with a time limit of two hours. A "-" denotes that no feasible solution could be found within the two hour time limit.

A problem instance is defined by the time period resolution, i.e. either monthly or annually, and in the case of an annual resolution whether an MMD grade tonnage curve was used or not and lastly, the size of the problem instance expressed in terms of the number of stoping blocks considered. Table 2 shows the results for solving the mine scheduling optimisation problem for a range of problem instances and a range of mineral prices. The column labelled "Mineral Price $(\times 1)$ " is an arbitrarily selected base price at which a high degree of selectivity can be observed. By increasing the mineral price by increments of $25 \%$ results are obtained for the columns labelled "Mineral Price $(\times 1.25)$ " up to "Mineral Price $(\times 2)$ ". An execution time limit of two hours were imposed on all problem instances and the objective function values listed under the columns labelled "NPV" are, therefore, the best net present values found within this time limit. The entries listed under the columns labelled "gap(\%)" are the optimality gaps found within the time limit, calculated 
as $\left|Z^{L}-Z^{U}\right| / Z^{U}$ with $Z^{L}$ and $Z^{U}$ the best lower and upper bounds respectively. Evidently an optimality gap of zero would imply that the specific problem instance was solved to optimality within the time limit.

All problem instances were solved using CPLEX 12.1 on an HP Elitebook 8540p with a Core i7 processor and 8GB RAM. The most notable result from Table 2 is the inability of CPLEX to generate feasible solutions within the set time limit for the RMSOP model when considering a monthly resolution and problem sizes exceeding 50 stoping blocks. In contrast, much larger problem instances could be solved with the LRMS model for all mineral price cases. Also very prominent is the inferior results produced by the RMSOP model with an annual time period resolution. Specifically, for the base mineral price $(\times 1)$, optimisation terminated optimally but with only a zero NPV for all problem sizes due to the inability of the RMSOP model to effectively harness the potential of selectivity as a result of the aggregation of information. In these cases the costs overshadowed revenue with the result that the most economical plan is to refrain from scheduling any mining activities. For other mineral price cases the RMSOP model with an annual time period resolution did provide non-zero NPV values that were, however, much lower compared to the corresponding results for both the RMSOP model with monthly periods as well as the LRMS model.

The benefits of employing an MMD grade tonnage curve in conjunction with the LRMS model is evident from the base mineral case $(\times 1)$. All NPV entries for the LRMS model exceed that of the other two cases where the RMSOP model was used for both a monthly and an annual time period resolution. For an increased mineral price the benefits of using the LRMS model diminishes to such an extent that for the $(\times 2)$ mineral price case no improvements in objective values are obtained over the result from the RMSOP model with monthly periods. However, for the said mineral price case, the RMSOP model with a monthly time period is still unable to produce any feasible integer solutions within the two hour time limit for problem sizes exceeding 50 stoping blocks, whereas with the LRMS model solutions could be computed.

In summary, the major benefit of using the LRMS model with MMD grade tonnage curves can be observed for problem instances where low profit margins drive selectivity and where the size of a problem instance in terms of the number of stoping blocks exceeds the computable capacity of a high resolution model formulation.

\section{Summary and conclusion}

The primary contribution of this paper is the introduction of the LRMS model which is based on a low resolution time discretisation, but with the capability of harnessing information captured within grade tonnage curves to allow selectivity on a micro level. The LRMS model outperforms high resolution discretised problem formulations specifically in low profit margin cases were selectivity is needed to boost profitability and in cases where the dimension of a problem instance is detrimental to computational feasibility. 


\section{References}

[1] Askari-Nasab H, Pourrahimian Y, Ben-Awuah E \& Kalantari S, 2011, Mixed integer linear programming formulations for open pit production scheduling, Journal of Mining Science, 47(3), pp. 338-359.

[2] Bley A, Boland N, Fricke C \& Froyland G, 2010, A strengthened formulation and cutting planes for the open pit mine production scheduling problem, Computers and Operations Research, 37(9), pp. 1641-1647.

[3] Caccetta L \& Hill SP, 2003, An application of branch and cut to open pit mine scheduling, Journal of Global Optimization, 27(2), pp. 349-365.

[4] Carlyle WM \& Eaves BC, 2001, Underground planning at Stillwater Mining Company, Interfaces, 31(4), pp. 50-60.

[5] Dantzig GB, 1960, On the significance of solving linear programming problems with some integer variables, Econometrica, 28, pp. 30-44.

[6] Epstein R, Goic M, Weintraub A, Catalán J, Santibáñez P, Urrutia R, Cancino R, Gaete S, Aguayo A \& Caro F, 2012, Optimizing long-term production plans in underground and open-pit copper mines, Operations Research, 60(1), pp. 4-17.

[7] Newman AM, Rubio E, Caro R, Weintraub A \& Eurek K, 2010, A review of operations research in mine planning, Interfaces, 40(3), pp. 222-245.

[8] Hustrulid WA \& Bullock RL, 2001, Underground mining methods: Engineering fundamentals and international case studies, Society for Mining Metallurgy and Exploration, Littleton (CO).

[9] Kazakidis V \& Scoble M, 2003, Planning for flexibility in underground mine production systems, Mining Engineering, 55(8), pp. 33-39.

[10] Little J, Nehring M \& Topal E, 2008, A new mixed integer programming model for mine production scheduling optimisation in sublevel stope mining, Proceedings of the 2008 Australian Mining Technology Conference, pp. 167-172.

[11] Markowitz HM \& Manne AS, 1957, On the solution of discrete programming problems, Econometrica, 25, pp. 84-110.

[12] Nehring M, Topal E \& Little J, 2010, A new mathematical programming model for production schedule optimization in underground mining operations, The Journal of the South African Institute of Mining and Metallurgy, 110(8), pp. 437-446.

[13] Newman AM \& Kuchta M, 2007, Using aggregation to optimize long-term production planning at an underground mine, European Journal of Operational Research, 176(2), pp. 1205-1218.

[14] Rahal D, Smith M, Van Hout G \& Von Johannides A, 2003, The use of mixed integer linear programming for long-term scheduling in block caving mines, Proceedings of the $31^{\text {st }}$ International Conference of the Application of Computers and Operations Research in the Mineral Industry Symposium, Cape Town, pp. 123-132.

[15] Rubio E \& Diering E, 2004, Block cave production planning using operation research tools, Proceedings of the 2004 MassMin Conference, Santiago, Chile, pp. 141-149.

[16] SARIN SC \& WeSt-HANSEn J, 2005, The long-term mine production scheduling problem, IIE Transactions, 37(2), pp. 109-121.

[17] Silva F \& Soares A, 2001, Grade Tonnage Curve: How far can it be relied upon?, Annual Conference of the International Association for Mathematical Geology, Cancún, pp. 1-11.

[18] Schulze M \& Zimmermann J, 2010, Scheduling in the context of underground mining, pp. 611-616 in Hu B, Morasch K, Pickl S \& Siegle M (Eds), Operations Research Proceedings 2010, Springer New York (NY).

[19] Smith M, Sheppard I \& Karunatillake G, 2003, Using MIP for strategic life-of-mine planning of the lead/zinc stream at Mount Isa Mines, Proceedings of the $31^{\text {st }}$ International Conference of the Application of Computers and Operations Research in the Mineral Industry Symposium, Cape Town, pp. $465-474$. 
[20] Topal E, 2008, Early start and late start algorithms to improve the solution time for long-term underground mine production scheduling, The Journal of the South African Institute of Mining and Metallurgy, 108(2), pp. 99-107.

[21] Whittle J, 1998, Four-X user manual, Melbourne: Whittle Programming Pty Ltd. 